\title{
Expression of MHC class II antigens by conjunctival epithelial cells in trachoma: Implications concerning the pathogenesis of blinding disease
}

\author{
D C W Mabey, R L Bailey, D Dunn, D Jones, J H D Williams, H C Whittle, M E Ward
}

\begin{abstract}
In an attempt to understand the pathogenesis of corneal changes and conjunctival scarring in trachoma, which are responsible for blindness in some 7 million people worldwide, the surface expression of major histocompatibility complex class II antigens on conjunctival epithelial cells was sought in patients with different clinical features of the disease. Class II expression was significantly associated with active (inflammatory) trachoma, but an independent association with corneal pannus or conjunctival scarring could not be shown. Longitudinal data suggest that class II expression may antedate clinically active disease and persist after it has resolved.
\end{abstract}

Department of

Clinical Sciences,

London School of

Hygiene and Tropical

Medicine, Keppel

Street, London WC1E

7HT

D C W Mabey

R L Bailey

Department of

Epidemiology and

Population Studies,

London School of

Hygiene and Tropical

Medicine

D Dunn

Medical Research

Council Laboratories,

Fajara, The Gambia

H C Whittle

University

Department of

Microbiology,

Southampton General

Hospital

MEWard

University

Department of

Pathology,

Southampton General

Hospital

D Jones

J H D Williams

Correspondence to:

Dr D C W Mabey

Department of Clinical

Sciences, London School of

Hygiene and Tropical

Hygiene and Tropical
Medicine, Keppel Street,

Medicine, Keppel Stree,
London WC1E 7HT.

Accepted for publication

6 December 1990
Trachoma remains a major cause of blindness second only to cataract in many parts of the world. It is believed to affect some 500 million people, of whom 7 million are blind. ${ }^{1}$ In its early stages trachoma is a chronic follicular conjunctivitis affecting principally the subtarsal conjunctiva, often with corneal disease in the form of punctate keratitis or vascular infiltration (pannus), which is occasionally so extensive as to impair vision. ${ }^{2}$ Chlamydia trachomatis can often be isolated from conjunctival swabs or scrapings at this stage. ${ }^{3}$

As the inflammatory changes subside they are frequently replaced by conjunctival scarring and fibrosis, which, if severe, may lead to distortion of the lid margin, entropion, and hence blindness secondary to prolonged corneal trauma. $C$ trachomatis can rarely be isolated from patients with scarring disease.

In endemic areas the early stages of trachoma are seen in young children, whereas conjunctival scarring is seen principally in adults in whom it seems to progress in severity over time. ${ }^{4}$

The pathogenesis of pannus and conjunctival scarring in trachoma remain unclear. There is evidence that in primate models both may be immunopathological in origin as they were only seen in animals which had been repeatedly infected or immunised. ${ }^{56}$ Trachoma vaccine trials in man showed no evidence that more severe pannus or scarring followed immunisation, although in two of these trials the incidence of trachoma was reported to be higher in the vaccinated group..$^{78} \mathrm{~A}$ migrant study suggested that conjunctival scarring does not progress in those who leave an endemic area, implying that continued exposure to $C$ trachomatis is a prerequisite. $^{9}$

It is not clear why scarring should progress in subjects from whom the organism cannot be isolated. It has been proposed that such patients may have latent chlamydial infections, and chlamydial antigen has been detected in a minority of patients with conjunctival scarring..$^{10}$ An alternative hypothesis is that an initial chlamydial infection may trigger certain immunological changes in the conjunctival epithelium, leading to chronic immunopathological damage.

The expression of class II major histocompatibility complex (MHC) molecules at the cell surface enables cells to present antigens to CD 4 positive lymphocytes. In healthy people class II expression is only found on specialised antigen presenting cells, B lymphocytes, activated $T$ lymphocytes and cells of certain epithelia, such as the small intestine, which frequently come in contact with foreign antigens. ${ }^{11}$ Expression of MHC class II antigens by other tissues has been linked to organ specific autoimmunity. ${ }^{12}$ We have explored the role of $\mathrm{MHC}$ class II expression by conjunctival epithelial cells in the pathogenesis of trachomatous pannus and scarring.

\section{Methods}

The study was carried out in the Gambian village of Jali. The epidemiology of trachoma in this village has been described previously. ${ }^{13}$

All patients were examined by the same observer (DCWM) with a $\times 4$ illuminated monocular loupe. Active trachoma was diagnosed on the basis of follicular and papillary changes in the subtarsal conjunctiva, according to the criteria of Dawson, Jones, and Tarizzo. ${ }^{1}$

Both follicles and papillary hypertrophy are graded on a scale of $0-3$. In the case of follicles grade 1 indicates the presence of follicles only at the lateral or medial margins. This is a common finding in this community and is not related to trachoma. Grades 2 and 3, which define active trachoma, indicate that there are at least five follicles in the central region. In the 
case of papillary hypertrophy grade 3 defines severe active trachoma and indicates hypertrophy great enough to obscure the conjunctival blood vessels over most of the subtarsal conjunctiva. Grades 1 and 2 indicate milder changes and do not of themselves define active trachoma.

The accuracy of clinical diagnosis was assessed by comparing the diagnosis obtained by DCWM and a team of ophthalmologists visiting Jali as part of a nationwide blindness and trachoma survey sponsored by the World Health Organisation. One hundred and sixty two subjects were examined by both DCWM and the WHO team, and agreement was achieved in $151(93 \%)$.

A total of 78 subjects were studied. All were children aged 17 years or less. The intention was to choose 40 subjects with active disease and 40 age and sex matched controls without active disease, but for logistic reasons specimens were only obtained from 38 subjects without active disease. Among the $\mathbf{4 0}$ children with active disease, 21 had pannus and 17 had conjunctival scarring. Among the children without active disease, pannus was present in three and conjunctival scarring in seven.

This study was approved by the joint Gambia Government/Medical Research Council ethical committee.

All subjects with clinically active trachoma were given a course of supervised treatment comprising a 10 day course of oral antibiotics (tetracycline or, in the case of children aged less than 6 years, erythromycin) and a four week course of $1 \%$ tetracycline ointment applied to both eyes twice daily.

COLLECTION AND PROCESSING OF SPECIMENS Swabs for detection of MHC class II expression were taken from the upper subtarsal conjunctiva by firmly rubbing it three to four times with a cotton wool tipped swab (MW 142, Medical Wire Co). The swab was immediately expressed into $1 \mathrm{ml}$ of transport medium (phosphate buffered saline $+5 \%$ bovine serum albumin), and stored on ice for up to hours, after which it was divided into five equal portions and cytocentrifuged. Cytocentrifuge preparations were air dried, fixed in acetone, and transported with desiccant at $-20^{\circ} \mathrm{C}$ to Southampton.

Swabs for detection of chlamydial antigen were collected as described above, at least one week before collecting swabs for MHC class II detection. Swabs and transport medium were

Table 1 Details of patients

\begin{tabular}{lclrl}
\hline Category & $n=$ & Sex $M / F$ & $\begin{array}{l}\text { Mean age } \\
\text { (years) }+(\text { range) }\end{array}$ & $\begin{array}{l}\text { MHC class II } \\
\text { positive (\%) }\end{array}$ \\
\hline $\begin{array}{l}\text { Active trachoma } \\
\text { with pannus }\end{array}$ & 40 & $23 / 17$ & $7 \cdot 5(1-17)$ & $28(70 \%)$ \\
without pannus & 21 & $12 / 9$ & $8 \cdot 4(1-17)$ & $17(81 \%)$ \\
with scarring & 19 & $11 / 8$ & $6 \cdot 6(1-14)$ & $11(58 \%)$ \\
without scarring & 17 & $6 / 11$ & $11 \cdot 3(5-17)$ & $13(76 \%)$ \\
Inactive & 23 & $17 / 6$ & $4 \cdot 8(1-15)$ & $15(65 \%)$ \\
$\quad$ with scarring & 38 & $20 / 18$ & $8 \cdot 9(1-15)$ & $10(26 \%)$ \\
$\quad$ without scarring & 77 & $4 / 3$ & $11 \cdot 4(6-15)$ & $3(43 \%)$ \\
$\begin{array}{l}\text { Chlamydial antigen } \\
\text { positive }\end{array}$ & 31 & $16 / 15$ & $8 \cdot 4(1-15)$ & $7(23 \%)$ \\
negative & 17 & & & $14(82 \%)$ \\
\hline
\end{tabular}

supplied by the manufacturer (Boots-Celltech Ltd), swabs being broken off in the medium, which were then stored on ice for up to six hours before being frozen at $-20^{\circ} \mathrm{C}$ and transported to Southampton.

\section{LABORATORY METHODS}

\section{Detection of MHC class II antigen}

Details of monoclonal antibodies used for the demonstration of MHC class II antigen on conjunctival epithelial cells present in the cytological preparations were as follows: TAL 1B5, specific for class II $\alpha$ chain, kindly supplied by Dr J Bodmer; and Tu 22, DQ specific, kindly supplied by Professor A Ziegler.

In all cases the alkaline phosphatase method of Cordell et al was used for the demonstration of antibody reactivity. ${ }^{14}$ Briefly, cytocentrifuge preparations were brought from $-20^{\circ} \mathrm{C}$ and allowed to dry at room temperature. Primary antibody was applied for 30 minutes, followed by three washes in TRIS-buffered saline (TBS, $\mathrm{pH} 7 \cdot 6)$. Subsequently, binding of the primary antibody was shown using an appropriate bridging anti-mouse reagent and complexes formed from alkaline phosphatase pre-mixed with a monoclonal antibody to alkaline phosphatase. Specimens were washed three times with buffer between each of these incubation stages. The final reaction product was shown using Fast Red TR to give precise localisation of the alkaline phospatase label in conjunction with haematoxylin counterstain. All primary and secondary reagents were used at previously determined working dilutions and appropriate controls were included for non-specific binding of the primary reagent and also of the reporter system.

Specimens were read blind by a single observer (MEW) and reported as negative or positive grade 1,2 , or 3 , depending on the number of positive cells and the intensity of staining. Grade 1 implied that less than $40 \%$ of cells expressed HLA DR, grade 2 that between $40 \%$ and $80 \%$ expressed HLA DR, and grade 3 that more than $80 \%$ of epithelial cells expressed HLA DR.

\section{Detection of chlamydial antigen}

Chlamydial antigen was assayed using the Boots-Celltech IDEIA amplified enzyme immunoassay, according to the manufacturer's instructions, as described previously. ${ }^{10}$

\section{Results}

The age and sex distribution of the different categories of patient are shown in tables 1 and 2. It was not possible to match subjects with and without conjunctival scarring by age, because this phenomenon is rare in children aged less than 10 years in Jali, and the number of children aged more than 10 years with active disease is small.

\section{MHC CLASS II EXPRESSION AND CLINICAI} FINDINGS

Equivalent results were obtained with the two monoclonal antibodies used. There was no difference between the sexes in the prevalence of 
Table 2 Age and sex distribution of active cases and controls

\begin{tabular}{lrrrrrrr}
\hline & \multicolumn{3}{l}{ Active trachoma } & & \multicolumn{3}{l}{ Inactive } \\
\cline { 2 - 3 } \cline { 6 - 7 } Age & Male & Female & Total & & Male & Female & Total \\
\hline $1-4$ & 8 & 4 & 12 & & 3 & 4 & 7 \\
$5-8$ & 12 & 1 & 13 & & 11 & 0 & 11 \\
$9-12$ & 2 & 5 & 7 & & 5 & 5 & 11 \\
$13-17$ & 1 & 7 & 8 & & 1 & 8 & 9 \\
Total & 23 & 17 & 40 & & 21 & 17 & 38 \\
\hline
\end{tabular}

MHC class II expression, which was seen in $21 / 44$ males $(48 \%)$ and $17 / 34$ females $(50 \%)$.

Of the 78 subjects studied, no MHC class II expression was observed in conjunctival epithelial cells from $40(51 \%)$. Grade 1 expression was observed in $17 / 40$ active cases $(42 \%)$, grade 2 in four (10\%), and grade 3 in seven (17\%). Among inactive cases, grade 1 expression was seen in six of $38(16 \%)$, grade 2 in three $(8 \%)$, and grade 3 in one $(3 \%)$. Overall class II expression was observed in conjunctival epithelial cells from 28 of $\mathbf{4 0}$ subjects with active trachoma $(70 \%)$, but in only 10 of 38 without active disease $(26 \%)$ (figure). This difference is highly significant $\left(\chi^{2}=13.2 ; \mathrm{p}<0.001\right)$.

The association between MHC class II expression and four clinical signs (follicular conjunctivitis, papillary hypertrophy, pannus and conjunctival scarring) is shown in table 3. As these are all continuous variables, results have been combined from subjects with and without active trachoma. Increasing follicular score, papillary hypertrophy, and pannus were all strongly associated with increased expression of MHC class II, but these signs of active disease were all closely interrelated. Logistic regression analysis suggested papillary hypertrophy score to be the strongest predictor of class II expression, with no additional effect of any other sign.

Table 4 shows the association between conjunctival scarring and class II expression in subjects aged 5 years and over; younger subjects have been excluded because scarring was not seen below this age, and results have been pooled for subjects with and without active trachoma for the reasons given above. Once again, papillary score is a strong confounding variable. Among those with a papillary hypertrophy score of $<2$, there is a suggestion that class II expression is more common in those with scarring (three of seven or $43 \% v$ one of 15 , or $7 \%$ ), but this difference is not significant ( $\mathrm{p}=0 \cdot 15$, Fisher's exact test).

MHC CLASS II EXPRESSION AND PRESENCE OF CHLAMYDIAL ANTIGEN

Chlamydial antigen was detected in conjunctival swabs from 14 of $40(35 \%)$ subjects with active trachoma. MHC Class II expression was observed in 12 of $14(86 \%)$ antigen positive subjects and in 16 of $26(62 \%)$ antigen negative subjects with active disease $\left(\chi^{2}=1.51\right.$; $\mathrm{p}>0 \cdot 1)$. Chlamydial antigen was also detected in three of $38(8 \%)$ subjects without active disease on clinical grounds. MHC class II expression was observed in two of these three. Overall, class II expression was found in 14 of $17(82 \%)$ subjects positive for chlamydial antigen, and in 24 of $61(39 \%)$ negative for chlamydial antigen $\left(\chi^{2}=8.2 ; \mathrm{p}<0.01\right)$. Logistic regression analysis suggested that the presence of chlamydial antigen had no significant additional effect over the above clinical signs on the expression of MHC class II.

\section{MHC CLASS II EXPRESSION AND EVOLUTION OF} DISEASE

As stated above, MHC class II expression was observed in 10 of 38 subjects without active disease on clinical grounds at the time the swab was taken. Of these 10 subjects, six $(60 \%)$ had active disease at the previous or subsequent survey (three at the survey eight months earlier and three at the survey 13 months later). Among 28 subjects without active disease
Conjunctival epithelial cells $(A)$ expressing $M H C$ class $I I$ antigen and $(B)$ not expressing class II antigen.

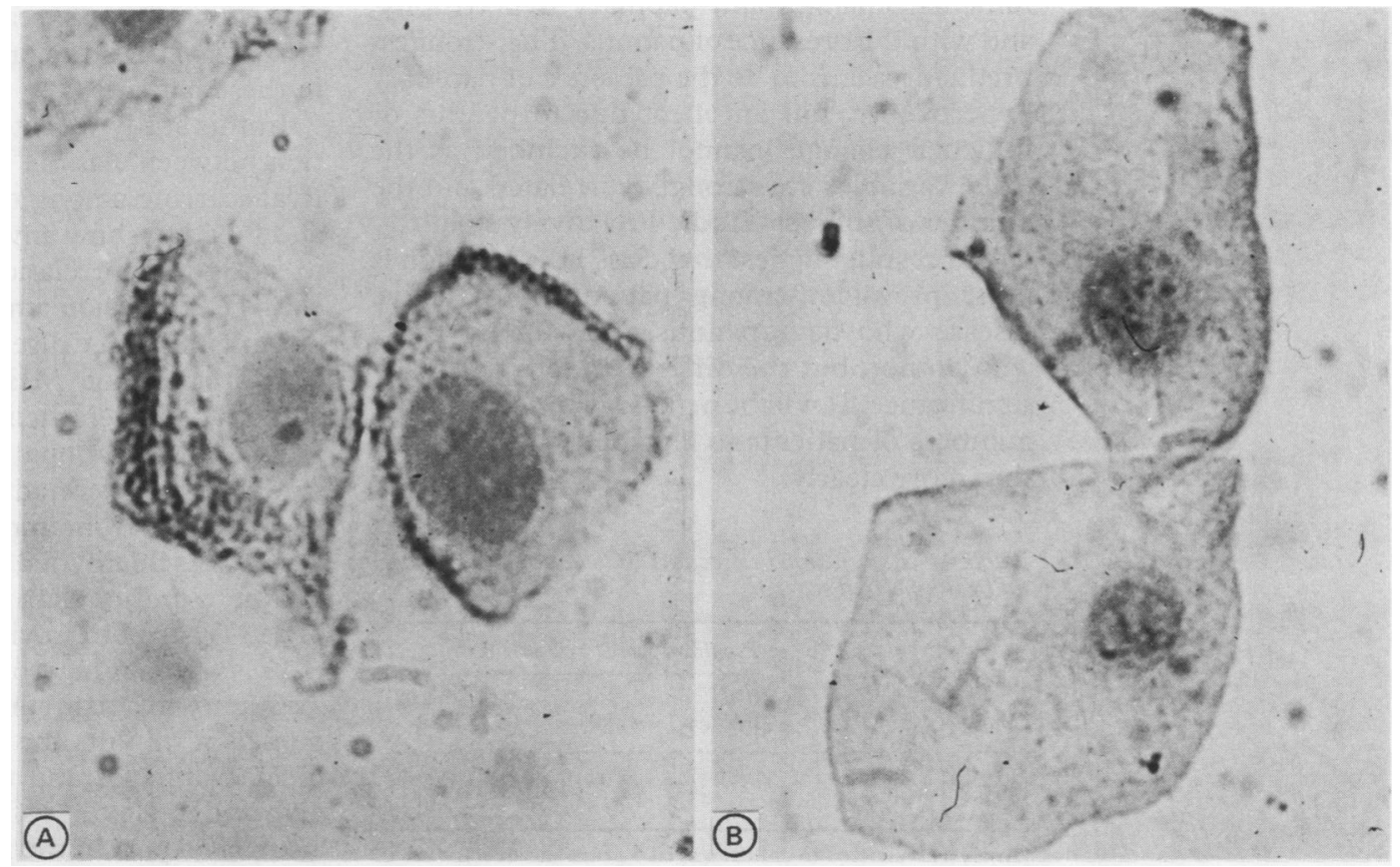


Table 3 MHC class II expression according to ocular signs

\begin{tabular}{|c|c|c|c|c|c|c|}
\hline \multirow[b]{2}{*}{ Sign } & \multicolumn{4}{|l|}{ Score } & \multicolumn{2}{|c|}{ Significance } \\
\hline & 0 & 1 & 2 & 3 & $\chi^{2}$ trend & pvalue \\
\hline $\begin{array}{l}\text { Follicles n (\%) } \\
\text { Papillary hypertrophy n (\%) } \\
\text { Pannus n (\%) } \\
\text { Conjunctival scarring n (\%) }\end{array}$ & $\begin{array}{l}5 / 19(26) \\
0 / 3(0) \\
19 / 54(54) \\
22 / 54(41)\end{array}$ & $\begin{array}{l}6 / 21(29) \\
14 / 44(32) \\
12 / 15(80) \\
15 / 22(68)\end{array}$ & $\begin{array}{l}20 / 29(69) \\
18 / 24(75) \\
5 / 6(83) \\
0 / 1(0)\end{array}$ & $\begin{array}{l}7 / 9(78) \\
6 / 7(86) \\
2 / 2(100) \\
1 / 1(100)\end{array}$ & $\begin{array}{r}11 \cdot 95 \\
15 \cdot 79 \\
11 \cdot 74 \\
2.95\end{array}$ & $\begin{aligned} 0.001 \\
<0.001 \\
0.001 \\
0.09\end{aligned}$ \\
\hline
\end{tabular}

negative for class II expression, only six (21\%) had active disease at the previous or subsequent survey. This implies that class II expression, rather than being a non-specific finding in this community, may precede active trachoma and persist after it has resolved, although the difference between the two groups does not quite achieve significance (six of $10 v$ six of 28 ; $\left.\chi^{2}=3.45 ; 0.1>p>0.05\right)$. We have observed persistent class II expression in the conjunctival epithelium of one subject on two occasions six months apart, his last recorded episode of active disease having been eight months before the first sample was taken.

\section{EFFECT OF TREATMENT ON EXPRESSION OF MHC} CLASS II

We studied the effect of the antimicrobial treatment of trachoma on the expression of MHC class II by the conjunctival epithelium in 21 subjects. Nine $(43 \%)$ of these subjects remained clinically active at the time the second specimen was taken (six months after the completion of treatment), although all were negative for chlamydial antigen. Fourteen $(67 \%)$ continued to express MHC class II:seven of nine with clinically active disease $(78 \%)$ and seven of $12(58 \%)$ who were clinically cured.

\section{Discussion}

We have shown that MHC class II antigens are commonly expressed by conjunctival epithelial cells in patients with active trachoma. Class II expression is correlated with the severity of follicular changes and papillary hypertrophy, and with the presence of pannus. The strongest predictor seems to be the presence of papillary hypertrophy, but an effect due to pannus or follicular changes cannot be excluded as the three variables are strongly correlated and the number of subjects studied relatively small.

Our results suggest that class II expression is more prevalent among patients with active disease who have pannus than among those who do not, but the difference does not reach significance. It will be necessary to study larger numbers of patients to elucidate this relationship more clearly.

Table 4 Association between $\mathrm{MHC}$ class II expression and conjunctival scarring

\begin{tabular}{llll}
\hline \multirow{5}{*}{ Papillary score } \\
\cline { 2 - 4 } Conjunctival scarring & 0,1 & 2,3 & \multicolumn{1}{l}{ All } \\
& $n(\%)$ & $n(\%)$ & $n(\%)$ \\
\hline No & $2 / 29(7)$ & $6 / 6(100)$ & $8 / 35(23)$ \\
Yes & $3 / 7(43)$ & $13 / 17(76)$ & $16 / 24(67)$ \\
\hline
\end{tabular}

(Analysis restricted to children aged 5 years and over).
Among those without active disease, MHC class II expression was more common in patients with active disease at a previous or subsequent survey. This may be due to misclassification by the clinical observer at the time the swab was taken, although this is perhaps unlikely in view of the adequate interobserver agreement achieved. It seems more likely that expression of class II antigen may precede the development of active disease and persist after it has resolved. We have shown that class II expression can persist for at least six months after adequate antimicrobial treatment of trachoma.

The persistent expression of MHC class II after the resolution of clinically active disease may have a part in the progression of conjunctival scarring which seems, on epidemiological grounds, to continue after the inflammatory process has subsided and $C$ trachomatis can no longer be isolated.

Longitudinal studies show that in some, but not all subjects with progressive conjunctival scarring, inapparent chlamydial infection can be shown by enzyme immunoassay of chlamydial antigen or associated tear antibody. ${ }^{15}$ It is possible that autoimmune mechanisms may be involved in the progression of pathological changes in subjects without evidence of persisting infection. Preliminary (unpublished) studies in our laboratory have shown the presence of antibody bound to conjunctival epithelial cells in patients with trachoma, and a correlation between bound antibody level and the titre of antichlamydial IgG antibody in ocular secretions, implying the presence of cross reacting epitopes between $C$ trachomatis and conjunctival epithelial cells in these patients.

In this study we set out to study the association between class II expression and conjunctival scarring among those with active disease, and failed to show any. It would be instructive to study the correlation between scarring and class II expression among older subjects without inflammatory disease.

Expression of MHC class II antigen by conjunctival epithelial cells is probably induced by gamma interferon (IFN); this cytokine is secreted by human $T$ helper lymphocytes from immune persons on incubation with chlamydial antigen ${ }^{16}$ and has been shown to induce class II expression in a variety of cell types both in vivo and in vitro. ${ }^{17-19}$

IFN gamma may play an important part in immunity to chlamydial infection, as in murine models it inhibits the growth of $C$ trachomatis in macrophages and other cell types both in vivo and in vitro..$^{20-22}$ On the other hand, it may also play a part in the immunopathology of the 
disease, either by inducing a state of chlamydial latency, with persistent release of sensitising chlamydial antigens from the infected cell, or by its recently proved cytotoxic effect on cells infected with $C$ trachomatis. ${ }^{23}$

The pathological importance of MHC class II expression by conjunctival epithelial cells in trachoma will need to be distinguished from the other effects of IFN described above. It may be that class II expression enables conjunctival epithelial cells to present antigen to local CD4 positive lymphocytes, leading to possible immunoregulatory defects; but experiments with corneal fibroblasts in vitro have shown that induction of class II expression by IFN gamma does not necessarily enable these cells to present antigen. Corneal fibroblasts treated with IFN gamma were not able to stimulate heterologous lymphocytes to divide and indeed inhibited autologous lymphocytes from acting as stimulators in the mixed lymphocyte re-

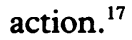

CD4 positive class II restricted cytotoxic T cells may play a part in the pathogenesis of trachoma through the lysis of conjunctival epithelial cells expressing MHC class II antigens. CD4 positive cytotoxic T cells raised against Mycobacterium bovis (BCG) have been shown to lyse monocytes expressing the mycobacterial 65 kilodalton heat shock protein (HSP); moreover, CD4 positive cells specific for the 65 kilodalton HSP also efficiently lysed autologous monocytes in the absence of antigen, suggesting that this protein may be an important trigger of autoimmunity. ${ }^{24}$ Interestingly, the mycobacterial 65 kilodalton HSP shares considerable sequence homology with the 57 kilodalton chlamydial heat shock protein that has been implicated in the pathogenesis of trachoma in subhuman primate models. ${ }^{25}$ Further study of cellular cytotoxic responses to chlamydial infection is likely to improve our understanding of the pathogenesis of chlamydial infections of the eye and genital tract. We are most grateful to Dr B M Greenwood for his support and
encouragement; to Dr R G Whitehead and the staff of the MRC encouragement; to Dr R G Whitehead and the staff of the MRC
Dunn Nutrition Unit, Keneba, for providing accommodation Dunn Nutrition Unit, Keneba, for providing accommodation
and laboratory facilities; to Bakary Dibba and Almami Janneh and laboratory facilities; to Bakary Dibba and Almami Janneh their good humoured cooperation.

This study was supported by the Edna McConnell Clark Foundation.

1 Dawson CR, Jones BR, Tarizzo ML. A guide to trachoma control. Geneva: World Health Organisation, 1981.

2 Schachter J, Dawson CR. Human chlamydial infections. Littleton, Mass: PSG Publishing, Inc, 1978.
3 Schachter J, Moncada J, Dawson CR, et al. Nonculture methods for diagnosing chlamydial infection in patients with trachoma: A clue to the pathogenesis of the disease. $J$ with trachoma: A clue to the path
Infect Dis 1988;158:1347-52.

4 Sowa S, Sowa J, Collier LH, Blyth W. Trachoma in a Gambian village. MRC Special Report Series. 1965: No. 308.

5 Wang S-P, Grayston JT, Alexander ER. Trachoma vaccine studies in monkeys. Am J Ophthalmol 1967;63:1615-30.

6 Wang S-P, Grayston JT. Pannus with experimenta trachoma and inclusion conjunctivitis agent infection of Taiwan monkeys. Am J Ophthalmol 1967;63:1133-45.

7 Woolridge RC, Grayston JT, Chang IH, Cheng KH, Yang $\mathrm{CY}$, Neave $\mathrm{C}$. Field trial of a monovalent and of a bivalent mineral oil vaccine in Taiwan school children. Am Ophthalmol 1967;63:1645-50.

8 Nichols RL, Bell SD, Murray ES, Hadad NA, Bobb AA Studies on trachoma. V. Clinical observations in a field trial of bivalent trachoma vaccine at three dosage levels in Saudi Arabia. Am J Trop Med Hyg 1966;15:639-47.

9 Detels R, Alexander ER, Dhir SP. Trachoma in Punjabi Indians in British Columbia. A prevalence study with comparions to India. Am J Epidemiol 1966;84:81-91.

10 Mabey DCW, Robertson JN, Ward ME. The detection of Chlamydia trachomatis by enzyme immunoassay in patients with trachoma. Lancet 1987;ii:1491-2.

11 Daar AS, Fuggle SV, Fabre JW, Ting A, Morris PJ. The detailed distribution of MHC class II antigens in normal human organs. Transplantation 1984;38:293-8.

12 Foulis AK. Class II major histocompatibility complex and organ specific autoimmunity in man. $J$ Pathol 1986;150: 5-11.

13 Bailey R, Osmond C, Mabey DCW, Ward ME. Analysis of the household distribution of trachoma in a Gambian
village using a Monte Carlo simulation procedure. Int $J$ village using a Monte Car
Epidemiol 1989;18:944-51.

14 Cordell JL, Falini B, Erber WN, et al. Immunoenzymatic labelling of monoclonal antibodies using immune complexes of alkaline phosphatase and monoclonal antialkaline phosphatase (APAAP complexes). $J$ Histochem Cytochem 1984;32:219-29.

15 Ward ME, Bailey R, Lesley A, Kajbaf M, Robertson J, Mabey D. Persisting inapparent chlamydial infection in a trachoma endemic community in The Gambia. Scand $J$ Infect Dis 1990;(Suppl 69):137-48.

16 Duc-Goiran $P$, Zernicki $L$. Interferons induced by Chlamydia trachomatis in human lymphocyte cultures. Biomedicine 1981;34:88-93.

17 Young E, Stark WJ. In vitro immunological function of human corneal fibroblasts. Invest Ophthalmol Vis $S c i$ human corneal

18 Kaplan G, Nusrat A, Sarno EM, et al. Cellular responses to the intradermal injestion of recombinant human gammainterferon in lepromatous leprosy patients. $\mathrm{Am} \mathrm{J} \mathrm{Pathol}$ 1987;128:345-53.

9 Fellous M, Nir U, Wallach D, Merlin G, Rubinstein M, Revel M. Interferon-dependent induction of mRNA for the major histocompatibility antigens in human fibroblasts and lymphoblastoid cells. Proc Natl Acad Sci USA 1982;79:3082.

20 Byrne GI, Krueger DA. Lymphokine-mediated inhibition of chlamydia replication in mouse fibroblasts is neutralised by anti-gamma interferon immunoglobulin. Infect Immun by anti-gamma

21 Zhong G, De La Maza LM. Activation of mouse peritoneal macrophages in vitro or in vivo by recombinant murine gamma interferon inhibits the growth of Chlamydia trachomatis serovar L1. Infect Immun 1988;56:3322-5.

22 Byrne GI, Grubbs B, Dickey TJ, Schachter J, Williams DM. A role for interferon in recovery from pneumonia due to Chlamydia trachomatis in the mouse. J Infect Dis 1987;156:993-6.

23 Byrne GI, Grubbs B, Marshall TJ, Schachter J, Williams DM. Gamma-interferon mediated cytotoxicity related to murine Chlamydia trachomatis infection. Infect Immun 1988;56:2023-7.

24 Ottenhof THM, Birhane Kale AB, Van Embden JDA Thole JER, Kiessling R. The recombinant $65 \mathrm{kD}$ heat shock protein of Mycobacterium bovis bacillus Calmetteshock protein of Mycobacterium bovis bacillus CalmetteGuerin $M$ tuberculosis is a target molecule for CD4 +
cytotoxic T lymphocytes that lyse human monocytes. $J$ cytotoxic T lymphocytes that

25 Morrison RP, Belland RJ, Lyng K, Caldwell HD. Chlamydial disease pathogenesis. The $57 \mathrm{kD}$ chlamydial hypersensitivity antigen is a stress response protein. $J$ Exp Med 1989;170:1271-83. 